\title{
Treatment outcomes of severe acute malnutrition and predictors of recovery in under-five children treated within outpatient therapeutic programs in Ethiopia: a systematic review and meta- analysis
}

Zebenay Workneh Bitew ${ }^{1 *}$, Ayinalem Alemu ${ }^{2}$ and Teshager Worku ${ }^{3}$

\begin{abstract}
Background: Severe acute malnutrition affects around 17 million under-five children in the world, of which the highest burden is accounted by Sub-Saharan Africa where Ethiopia is found. Though there are few individual, inconsistent and inconclusive studies, there is no nationally representative study on treatment outcomes of SAM in outpatient therapeutic feeding programs of Ethiopia. This study aimed at estimating the pooled treatment outcomes and predictors of recovery rate among under- five children with SAM in Ethiopia.

Methods: Electronic databases (PubMed, Medline (EBSCOhost), EMBASE (Elsevier), CINAHL (EBSCOhost), web of science, Scopus, Science Direct and Food Science and Technology Abstracts (FSTA)), and grey literature sources (Google scholar, Mednar, World Cat and google) were used to retrieve articles. The random effect model was used to estimate the pooled treatment outcomes. Hazard ratios were used to determine the predictors of recovery rate. Cochran's $\mathrm{Q}, \mathrm{I}^{2}$, and univariate Meta regression were done for heterogeneity. Begg's \& Egger's tests were used for publication bias.

Results: Nineteen articles with a total number of 23,395 under-five children with SAM were used for this metaanalysis. The pooled recovery, death, defaulter and non-recovery rates were $70 \%(95 \% \mathrm{Cl}: 64,76), 2 \%(95 \% \mathrm{Cl}: 1,2)$, $10 \%$ (95\%Cl: 7, 12), 15\% (95\% Cl: 10, 20), respectively. Diarrhea ( $\mathrm{HR}=0.8,95 \% \mathrm{Cl}: 0.75,0.94)$, no edema (HR $=0.41$, $95 \%$ Cl: $0.33,0.50)$ and amoxicillin ( $H R=1.81,95 \% \mathrm{Cl}: 1.18,2.44)$ were independent predictors of recovery rate of children with SAM in Ethiopia. Publication year was found to be the potential source of heterogeneity between included studies.

* Correspondence: zedo2015@gmial.com

'Department of Pediatric Nursing, St. Paul's Hospital Millennium Medical College, School of Nursing, Addis Ababa, Ethiopia

Full list of author information is available at the end of the article

C The Author(s). 2020 Open Access This article is licensed under a Creative Commons Attribution 4.0 International License, which permits use, sharing, adaptation, distribution and reproduction in any medium or format, as long as you give appropriate credit to the original author(s) and the source, provide a link to the Creative Commons licence, and indicate if changes were made. The images or other third party material in this article are included in the article's Creative Commons licence, unless indicated otherwise in a credit line to the material. If material is not included in the article's Creative Commons licence and your intended use is not permitted by statutory regulation or exceeds the permitted use, you will need to obtain permission directly from the copyright holder. To view a copy of this licence, visit http://creativecommons.org/licenses/by/4.0/. The Creative Commons Public Domain Dedication waiver (http://creativecommons.org/publicdomain/zero/1.0/) applies to the data made available in this article, unless otherwise stated in a credit line to the data. 


\begin{abstract}
(Continued from previous page)
Conclusion: The treatment outcomes of children with SAM from outpatient therapeutic feeding programs of Ethiopia are lower than the sphere guidelines, WHO and national recommendations. Diarrhea and no edema antagonized the recovery rate of children, while amoxicillin enhanced the recovery rate of children from SAM. Community health workers need to be trained. Especial attention should be given while treating children with diarrhea and severe wasting. Community mobilization is also recommended to improve community awareness about the therapeutic foods.
\end{abstract}

Keywords: Survival, Severe malnutrition; cure rate, Determinants, Outcomes; regions of Ethiopia

\section{Background}

Severe acute malnutrition is defined as very low weight for height/ length $(<-3 \mathrm{z}$ score of the median world health organization growth standards or presence of bilateral edema or Mid Upper Arm Circumference $<115$ $\mathrm{mm}$ for a child $\geq 6$ months age [1]. The magnitude of SAM remains high with an estimated 17 million underfive children were victims of SAM in 2016, of which the majority were from Africa and Asia where Ethiopia is found $[2,3]$. Of these, around 16.6 million children were affected by severe wasting [4]. The unwanted treatment outcomes of SAM also remain high throughout the globe which escalates the risk of death by 12 times as compared to well-nourished children [5]. Despite global progress in the number of children treated for SAM (from 1.1 million in 2009 to 4.4 million in 2017), only one in four children receives treatment [4].

Children with SAM get treatment either in the inpatient units or in the outpatient therapeutic feeding programs. Outpatient therapeutic feeding program (OTP) is part community-based management of acute malnutrition (CMAM) for children with uncomplicated severe acute malnutrition (SAM) [6]. The program services include diagnosis and provision of ready-to use therapeutic foods (RUTF) every week for 2 months; supplementation of medications like amoxicillin, folic acid, vitamin-A, measles vaccine and deworming [7].

Prior to the implementation OTP in Ethiopia, children with SAM were treated in the inpatient units with its many limitations $[8,9]$. Limited coverage and impact, costliness, cross infections, and high rate of mortality rate were some of the challenges contributing to inefficacy of inpatient management of SAM [10, 11]. Due to those limitations, OTP was endorsed as a health care system since 2005 [12] to treat children with uncomplicated SAM after the pilot that was tested since 2000 [13]. This is because early detection and OTP management is the cornerstone in the modern management of SAM that can limit the number of children in need of inpatient care [14]. Community based management can also help for early detection of children with SAM which could enhance early recovery [15]. Besides, treating
SAM children in OTP centers could minimize the costs spent in inpatient based programs [16, 17].

In spite of the fact that OTP has been implemented in Ethiopia from the inception of the program, the recovery rate of under-five children with SAM still remained unacceptably high. This is substantiated by the original studies conducted in different regions of the country with the recovery rates ranging from $32.7 \%$ [18] to $92.7 \%$ [19]. The recovery rates in most of the original studies $[12,18,20-28]$ were below the sphere standard [29]. The death rates also remain significantly high in some OTP centers of the country reaching as high as $14 \%$ [25]. Moreover, a remarkable variation is seen in the defaulting rates and nonrecovery rates. The defaulting rate ranged from $1.67 \%$ [23] to $25.2 \%$ [12] and the non-recovery rate ranged from to $2 \%$ [20] to $61.13 \%$ [18]. These inconsistent and inconclusive findings implied as there are unfinished tasks in the management process of SAM in Ethiopia. These inconsistencies could be attributed by multiple challenges during the implementation process of OTP. The common challenges that made the country not on the course of meeting the goals include; food sharing, trading of RUTFs as commodity, high cost of standard RUTFs, stigma associated with RUTFs use, lack of antibiotics, inappropriate exit from the program, and disliking the taste of RUTFs [7, 30]. In-addition, the determinants of treatment outcomes, particularly the predictors of recovery rate are not addressed comprehensively. Few studies [21, 24-26, 31-35] revealed that edema, diarrhea, deworming, giving antibiotics, vitamin A supplementation, and distance from OTP centers and age were predictors of recovery of under-five children with SAM in OTPs of Ethiopia.

Therefore, the main purpose of this systematic review and meta-analysis is determining treatment outcomes and predictors of recovery rate among under-five children with SAM in the outpatient therapeutic feeding programs in Ethiopia. The findings could help policy makers, stakeholders, and community health workers for the appropriate management of SAM at the OTPs. 


\section{Methods}

\section{Searching strategies}

In this systematic review and meta-analysis, the preferred reporting items for systematic review and metaanalysis (PRISMA) [36] was followed to write the whole document. All possible studies were retrieved comprehensively from the reputable databases (PubMed, Medline (EBSCOhost), EMBASE (Elsevier), CINAHL (EBSCOhost), web of science, Scopus, Science Direct and Food Science and Technology Abstracts (FSTA)) and grey literature sources (Google scholar, Mednar, World Cat and google). The reference lists of included studies were also checked and searched accordingly. Two author (ZWB \& TW) searched studies independently using the key terms: (a) population (infants, toddlers, preschoolers, under-five children); (b) exposure (severe acute malnutrition, SAM, severe malnutrition, protein energy malnutrition, PEM, uncomplicated severe acute malnutrition) (c) outcome (recovery, survival, cure rate, death, non-recovery, non-responder, transfers); (d) study design (cohort, cross-sectional, prevalence, epidemiology, observational); (e) study setting (outpatient treatment program, OTP, community based management of acute malnutrition, CMAM, health posts, health centers) and (f) location (Ethiopia, regions of Ethiopia, parts of Ethiopia). The Boolean search operators such as "OR", "AND", "AND/OR" were used during the searching process. The appropriateness of key terms was verified before the actual search was conducted. Literature searches were limited to, articles conducted in the English language. The EndNote X8 reference manager was used to manage the literatures. In this study, studies conducted from 2007 to January 20, 2020 were included.

\section{Eligibility criteria \\ Inclusion criteria}

The two investigators (ZWB \& AA) independently assessed the contents of each of the included studies and articles that met the following criteria were included in the final analysis.

\section{Population}

Studies, which were done among under-five children, were included.

\section{Study setting}

The studies conducted in areas where OTPs are implemented (health posts, health centers) were considered.

\section{Study area}

Studies conducted in Ethiopia were included.

\section{Study design}

Original articles which were conducted both in crosssectional and cohort study designs measuring treatment outcomes and associated factors were considered.

\section{Language}

Studies conducted in the English language were considered.

\section{Publication condition}

Studies fulfilling the predefined criteria (published or unpublished studies) were included.

\section{Exclusion criteria}

Two authors (ZWB \&AA) did data extraction blindly and independently after reviewing the abstracts and full texts of included studies. In addition, methodological qualities of studies were assessed by three authors (ZWB, AA, \& TW) independently. We excluded studies that were difficult to access the full text after failing to communicate the corresponding authors.

\section{Data abstraction and critical appraisal of the studies}

Structured and pre-tested data extraction checklist was used to extract the data by two authors (ZWB \& AA). The terms included in the extraction checklist were; the name the first author \& publication year, study region, study design, study period, study setting, age of study subjects, sample size, treatment outcomes (recovery, death, defaulting, non-recovery, unknown), median recovery days and predictors of recovery. The third author (TW) actively involved in resolving disagreements between two authors. The qualities of the studies were assessed using the Joana Briggs Institute checklists of cross-sectional and cohort studies [37]. Critical appraisal was done by two authors (ZWB \& AA), independently and blindly. The tools have Yes/No questions and 1 was given for Yes and 0 for others. The scores were summed up and changed to percentages. Studies with $>50 \%$ quality score were included in the meta-analysis (See Additional file 3). The mean scores of the two reviewers were used for final decision of inclusion of the studies in this systematic review and meta-analysis. During critical appraisal, the third author (TW) played a crucial role in solving the incongruities between two authors. The asymmetry of the funnel plot and/or statistical significance of Egger's regression test $(p<0.05)$ [38] were considered as indicators of publication bias.

\section{Operationalization of the outcomes}

The primary outcome of this study is the recovery rate of under-five children from severe acute malnutrition who were treated from OTP centers of Ethiopia. It was computed by dividing the number of children recovered 
to the total sample and multiplying it by 100 . The second outcome was the predictors of recovery using the hazard ratios from the included studies. The other outcomes were death rate, defaulter rate, non-recovery rate and all were calculated in the same approach recovery rate was calculated. The binomial distribution formula was used to compute the standard errors for each original study. In the current study, children who didn't respond to the therapeutic foods, those who were referred to the inpatient units due to medical complication and those transferred out before the discharge date were considered as non-recovered cases.

\section{Data analysis and assessment of certainty in the findings}

The data were extracted using an extraction checklist prepared using Microsoft excel 2016 (Table 1). Data were imported into STATA Version 15 (STATA Corporation, College Station Texas) software for analysis of the pooled estimates of recovery rates, death rates, defaulter rates, non-recovery rates and predictors of recovery rate of under-five children with SAM. Forest plot and summery tables were used to report meta-analyses results. The pooled estimates of outcomes and predictors were analyzed with 95\% CI. Heterogeneity among studies was explored by using forest plot and $\mathrm{I}^{2}$ test and Cochrane Q statistics [39]. The $\mathrm{I}^{2}$ values of 25,50 and $75 \%$ were interpreted as low, medium and high heterogeneity, respectively. In this study, the presence of heterogeneity was declared and justified when was $\mathrm{I}^{2} \geq 50 \%$ and a $P$ value of $<0.05$. The statistical tests pinpointed that there was heterogeneity [40] among the studies $\left(\mathrm{I}^{2}=98.7 \%, P=0.000\right)$. Random and fixed effect models were used interchangeably in the analyses. Since there was no a significant difference were observed, a random effects model was used to estimate the Der Simonian and Laird's pooled effect size of recovery rate [41, 42].

To identify the possible sources of heterogeneity, meta-regression analysis was done using the sample size and publication year as the possible source of variability. However, sample size was found to be statistically insignificant $(P=0.064)$ and publication year was found to be the possible source of variation $(P<0.001)$ (Table 2). Funnel plot was drawn using recovery rate and standard error of recovery rate it revealed as there is a publication bias (Fig. 3). The possible source of publication bias was also objectively examined using Egger's weighted correlation and Begg's regression tests [43]. The result showed that as there is publication bias $(P=0.036)$ in the Egger test and Begg's test was found to be insignificant $(P=$ $0.944)$. Hence, the pooled estimate of recovery rate was determined using Duval and Tweedie's Trim and Fill analysis in the Random-effects model. In addition, subgroup analysis was done using the study region and study year. This is done to minimize the random variations between the point estimates of the included studies.

\section{Results \\ Selection of eligible studies}

In the first search, 694 studies were found from electronic databases and grey literature sources. Of the total studies, 150 were duplicated files and 455 studies were removed after screening based on titles and abstracts. The full texts of 89 articles were reviewed. Finally, 19 articles [12, 18-28, 31-35, 44, 45] were included in the final analysis of this systematic review and meta-analysis (Fig. 1).

\section{Characteristics of the original studies}

The details of all the included studies are clearly summarized in Table 1. Cohort [18, 20, 21, 24-27, 31-35, $44,45]$ and cross-sectional [12, 19, 22, 23, 28] studies were included in this study. Coming to the regional distribution of studies, most of the studies were done in Southern Nations, Nationalities and Peoples of Ethiopia (SNNP) [18, 19, 22-24, 26, 32, 33, 44, 45]. While, three were form Tigray region $[20,25,35]$ and two of the studies were conducted in Amhara region [21, 27]. The others were done DireDawa administration [31], Afar region [34] and Oromia region [28]. One study was conducted from patient records in Addis Ababa, SNNP and Oromia [12]. The studies were done with review of documents form health centers and health posts and the sample sizes ranged from 163 in Oromia region [28] to 12,316 in SNNP [44]. The maximum recovery rate (92.7\%) was recorded in SNNP and the minimum one (55\%) was from the study done in Oromia region, Addis Ababa and SNNP [12]. In this systematic review and meta-analysis, a total of 23,395 under-five children with SAM who were treated in OTPs of different regions of Ethiopia were included. The included studies were conducted from 2007 to 2019. Regarding the quality scores of studies, eleven of them were classified under high quality, whereas, seven and one of them were classified under medium and low qualities, respectively. Moreover, the median recovery times were explored and only seven studies $[21,26,31-35]$ reported this. The median recovery time ranged from 5 weeks (interquartile range: 4.67 , 5.33) [33] to 8.7 weeks (interquartile range: 5,14 ) [31].

\section{Treatment outcomes of children with SAM in Ethiopia}

A total of 19 studies [12, 18-28, 31-35, 44, 45] were used to compute the pooled estimate of recovery rate of under-five children with SAM who were treated in the OTPs of Ethiopia. The recovery rate was found to be $70 \%$ (95\% CI: 64, 76, $\mathrm{I}^{2}=98.69 \% \& P=0.000$ ) (Fig. 2). The $\mathrm{I}^{2}$ statistic shows significant heterogeneity among studies. Due to this, the possible sources were checked 
Table 1 Summary of 19 included studies on treatment outcomes of SAM among under-five children admitted to outpatient therapeutic feeding programs in Ethiopia

\begin{tabular}{|c|c|c|c|c|c|c|c|c|c|c|c|c|}
\hline $\begin{array}{l}\text { Author, } \\
\text { Publication } \\
\text { year }\end{array}$ & $\begin{array}{l}\text { Study } \\
\text { region }\end{array}$ & $\begin{array}{l}\text { Study } \\
\text { design }\end{array}$ & $\begin{array}{l}\text { Study } \\
\text { period }\end{array}$ & $\begin{array}{l}\text { Study } \\
\text { setting }\end{array}$ & $\begin{array}{l}\text { Age } \\
\text { (months) }\end{array}$ & $\begin{array}{l}\text { Sample } \\
\text { size }\end{array}$ & $\begin{array}{l}\text { Median recovery } \\
\text { time in weeks } \\
\text { (IQR) }\end{array}$ & $\begin{array}{l}\text { Recovery } \\
\text { rate N (\%) }\end{array}$ & $\begin{array}{l}\text { Death } \\
\text { rate N } \\
(\%)\end{array}$ & $\begin{array}{l}\text { Defaulter } \\
\text { rate N (\%) }\end{array}$ & $\begin{array}{l}\text { Non- } \\
\text { recovery } \\
\text { N (\%) }\end{array}$ & $\begin{array}{l}\text { Quality } \\
\text { Scores }^{a}\end{array}$ \\
\hline $\begin{array}{l}\text { Degebasa, } \\
2017 \text { [1] }\end{array}$ & Tigray & Cohort & $\begin{array}{l}2011- \\
2015\end{array}$ & $\mathrm{HC}$ & $6-59$ & 2009 & - & $1406(70)$ & $1(0.06)$ & 74 (3.66) & 40 (1.99) & 7 \\
\hline $\begin{array}{l}\text { Mamo, } 2019 \\
\text { [2] }\end{array}$ & Amhara & Cohort & 2017 & $\mathrm{HC}$ & $6-59$ & 389 & $5.5(3.5,7.5)$ & $254(65.3)$ & - & $17(4.37)$ & $41(10.54)$ & 10 \\
\hline $\begin{array}{l}\text { Boltena, } \\
2008 \text { [3] }\end{array}$ & SNNP & $\begin{array}{l}\text { Cross } \\
\text { sectional }\end{array}$ & 2008 & $\mathrm{HC}$ & $<59$ & 355 & - & $329(92.7)$ & $11(3)$ & $7(2)$ & $8(2.3)$ & 6 \\
\hline $\begin{array}{l}\text { Kabalo, } \\
2017 \text { [4] }\end{array}$ & SNNP & $\begin{array}{l}\text { Cross } \\
\text { sectional }\end{array}$ & 2014 & $\mathrm{HP}$ & $6-59$ & 776 & - & $504(64.9)$ & $9(1.2)$ & $17(2.2)$ & $246(31.7)$ & 8 \\
\hline $\begin{array}{l}\text { Yebyo, } 2013 \\
\text { [5] }\end{array}$ & Tigray & Cohort & $\begin{array}{l}2008- \\
2012\end{array}$ & $\begin{array}{l}\mathrm{HC} \& \\
\mathrm{HP}\end{array}$ & $6-59$ & 628 & - & $\begin{array}{l}388 \\
(61.78)\end{array}$ & $\begin{array}{l}87 \\
(13.85)\end{array}$ & 19 (3.02) & $56(8.91)$ & 10 \\
\hline $\begin{array}{l}\text { Kabalo,2018 } \\
{[6]}\end{array}$ & SNNP & Cohort & $\begin{array}{l}2014- \\
2015\end{array}$ & $\mathrm{HP}$ & $0-59$ & 582 & - & $396(68)$ & $6(1.57)$ & $10(1.72)$ & $170(29.2)$ & 9 \\
\hline $\begin{array}{l}\text { Kabalo,2016 } \\
\text { [7] }\end{array}$ & SNNP & $\begin{array}{l}\text { Cross } \\
\text { sectional }\end{array}$ & 2015 & $\mathrm{HP}$ & $<59$ & 600 & - & $396(66)$ & $4(0.7)$ & $10(1.7)$ & - & 8 \\
\hline $\begin{array}{l}\text { Shanka, } \\
2015 \text { [8] }\end{array}$ & SNNP & Cohort & $\begin{array}{l}2011- \\
2013\end{array}$ & $\begin{array}{l}\mathrm{HC} \& \\
\mathrm{HP}\end{array}$ & $<59$ & 711 & $7.14(5.28,8.14)$ & $522(67.7)$ & $13(1.8)$ & 175 (24.6) & $13(1.83)$ & 10 \\
\hline $\begin{array}{l}\text { Atnafe, } \\
2019 \text { [9] }\end{array}$ & Dre Dawa & Cohort & $\begin{array}{l}2013- \\
2016\end{array}$ & $\begin{array}{l}\mathrm{HC} \& \\
\mathrm{HP}\end{array}$ & $6-59$ & 713 & $8.7(5,14)$ & $569(79.8)$ & $4(0.6)$ & $80(11.2)$ & $42(5.9)$ & 10 \\
\hline $\begin{array}{l}\text { Mengesha, } \\
2016 \text { [10] }\end{array}$ & SNNP & Cohort & $\begin{array}{l}2008- \\
2009\end{array}$ & $\mathrm{HP}$ & $6-59$ & 348 & 6 & $274(78.7)$ & - & - & $74(21.3)$ & 9 \\
\hline $\begin{array}{l}\text { Teshome, } \\
2019 \text { [11] }\end{array}$ & SNNP & Cohort & 2015 & $\mathrm{HP}$ & $6-59$ & 216 & $5(4.67,5.33)$ & 172 (79.6) & - & $8(3.7)$ & $36(16.7)$ & 9 \\
\hline $\begin{array}{l}\text { Liben, } 2019 \\
\text { [12] }\end{array}$ & Afar & Cohort & 2017 & $\begin{array}{l}\mathrm{HC} \& \\
\mathrm{HP}\end{array}$ & $6-59$ & 286 & $7(2.7,8.1)$ & $238(83.2)$ & $8(2.8)$ & $18(6.3)$ & $22(7.7)$ & 10 \\
\hline $\begin{array}{l}\text { Tadesse, } \\
2018 \text { [13] }\end{array}$ & SNNP & Cohort & 2011 & $H P$ & $6-59$ & 759 & - & $248(32.7)$ & $17(2.2)$ & $18(2.4)$ & $464(61.1)$ & 6 \\
\hline $\begin{array}{l}\text { Beletew, } \\
2019 \text { [14] }\end{array}$ & Amhara & Cohort & $\begin{array}{l}2016- \\
2019\end{array}$ & $\mathrm{HP}$ & $0-59$ & 600 & - & $390(65)$ & $12(2)$ & $96(16)$ & $102(17)$ & 8 \\
\hline $\begin{array}{l}\text { Yorra, } 2016 \\
\text { [15] }\end{array}$ & SNNP & Cohort & $\begin{array}{l}2013- \\
2015\end{array}$ & $\begin{array}{l}\mathrm{HC} \& \\
\mathrm{HP}\end{array}$ & $6-59$ & 602 & - & $414(68.8)$ & $8(1.3)$ & $145(24.1)$ & $21(3.5)$ & 7 \\
\hline $\begin{array}{l}\text { Massa, } 2016 \\
{[16]}\end{array}$ & Tigray & Cohort & 2012 & $\begin{array}{l}\mathrm{HC} \& \\
\mathrm{HP}\end{array}$ & $6-59$ & 332 & $7(4,8)$ & $255(76.8)$ & $2(0.6)$ & $58(17.5)$ & $17(5.1)$ & 10 \\
\hline $\begin{array}{l}\text { Mokgatle, } \\
2015 \text { [17] }\end{array}$ & Oromia & $\begin{array}{l}\text { Cross } \\
\text { sectional }\end{array}$ & 2010 & $\mathrm{HP}$ & $6-59$ & 163 & - & $114(69.9)$ & - & $36(22.1)$ & - & 6 \\
\hline $\begin{array}{l}\text { Teferi,2009 } \\
\text { [18] }\end{array}$ & SNNP & Cohort & $\begin{array}{l}2003- \\
2005\end{array}$ & $\begin{array}{l}\mathrm{HC} \& \\
\mathrm{HP}\end{array}$ & $0-59$ & 12,316 & - & $9871(80)$ & $217(2.5)$ & - & - & 8 \\
\hline $\begin{array}{l}\text { Belachew, } \\
2007 \text { [19] }\end{array}$ & $\begin{array}{l}\text { AA, SNNP } \\
\& \text { Oromia }\end{array}$ & $\begin{array}{l}\text { Cross } \\
\text { sectional }\end{array}$ & 2006 & $\mathrm{HC}$ & $<59$ & 1010 & - & $554(55)$ & $4(0.4)$ & $255(25.2)$ & 197 (19.5) & 6 \\
\hline
\end{tabular}

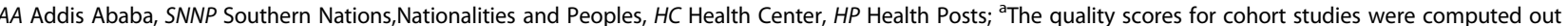
of 11 indicators while for cross sectional studies from 8 indicators; IQR interquartile range

Table 2 Factors associated with heterogeneity of recovery rate of children with SAM in Ethiopia (univariate meta-regression)

\begin{tabular}{lll}
\hline Variables & Coefficient & $P$-value \\
\hline Publication Year & 2015.4 & $<0.001$ \\
Sample Size & 1231 & 0.064 \\
\hline
\end{tabular}

using univariate meta-regression analysis by using publication year and sample size (Table 2). Sample size was found to be insignificantly associated $(P=0.064)$ and publication year was found to be the possible source of variation $(P<0.001)$. Publication bias was checked using funnel plots which showed the possibility of bias (Fig. 3). The presence of publication bias was confirmed by objective measures using Begg's and Egger's test. The Egger's test revealed significant publication bias $(P=$ $0.036)$, but Begg's test was found to be insignificant $(P=$ 


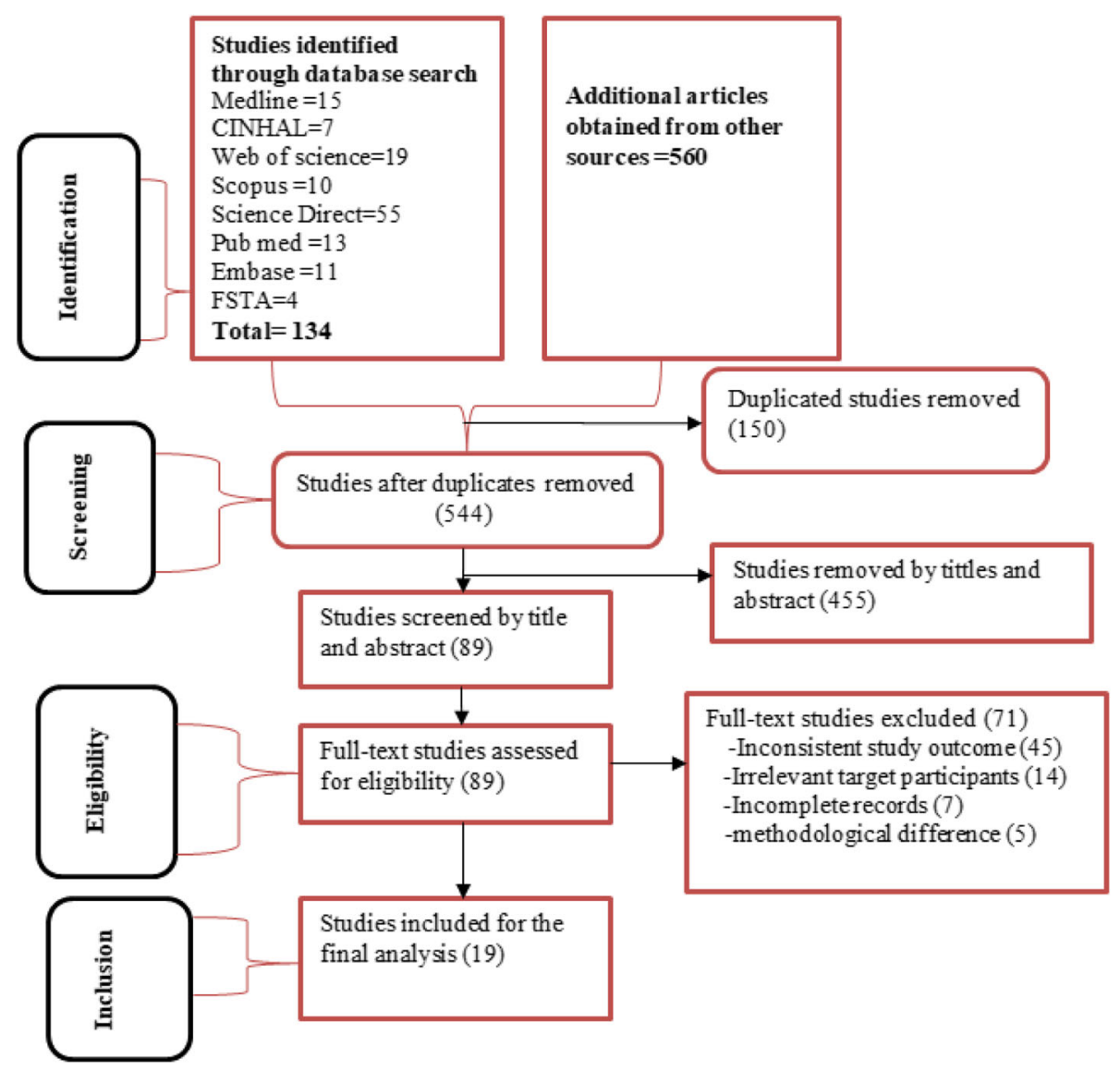

Fig. 1 The PRSIMA flow chart showing the selection process of studies

0.944). Therefore, Trim and Fill analysis was done to adjust the final pooled recovery rate of children with SAM who were treated in OTPs of Ethiopia.

The other treatment outcomes were death rate, defaulter rate, and non-recovery rates. All were computed based on the random effect models due to the presence of significant heterogeneity. In this review, 15 studies $[12,18-20,22-27,31,34,35,44,45]$ were used to compute the pooled estimate of death rate. The minimum $(0.05 \%)$ [20] and the maximum (13.85\%) [25] death rates were reported from the studies conducted in Tigray region. In this meta-analysis, the pooled death rate was found to be $2 \%$ (95\% CI: $1,2, \mathrm{I}^{2}=95.76 \%, P=0.000$ ) (Fig. 4).

To compute the pooled estimates of defaulter rate of children with SAM from OTPs of Ethiopia, 17 studies $[12,18-28,31,33-35,45]$ were used. The minimum defaulting rate $(1.67 \%)$ was reported from a study conducted in SNNP [23] and the maximum one (25.2\%) was from a study done in 2007 [12]. In our study, the defaulter rate was $10 \%\left(95 \% \mathrm{CI}: 7,12, \mathrm{I}^{2}=97.95 \%, P=\right.$ 0.000) (Fig. 5). In the present study, the pooled estimate of non-recovery rate was computed from 16 studies [12,
18-22, 24-27, 31-35, 45]. From the included studies, a study conducted at the SNNP revealed that majority $(61.13 \%)$ of children non-recovered and the lowest (1.83\%) non-recovery rate was reported from a study done in the other part of SNNP. Non-recovery rate was 15\% (95\% CI: $10,20, \mathrm{I}^{2}=99.2, P=0.000$ ) (Fig. 6).

\section{Subgroup analysis}

As it is illustrated in Table 3, subgroup analysis was done using publication year, study region and study settings. This is done to explore the possible sources of heterogeneity of the included studies. Accordingly, six studies were done from 2007 to 2015 with the recovery rate $72 \%$ (95\% CI: 62,82$)$ and the pooled recovery rate was lower in studies conducted after 2015 in Ethiopia (69, 95\% CI: 62, 76). In accordance with the region where the studies were done, the highest recovery rate (72, 95\% CI: 57, 87) was recorded form regions which were classified as others (Oromia, Afar, Dire Dawa, and (Oromia, Addis Ababa \& SNNP)). The second higher recovery rate $(71,95 \%$ CI: 61,80$)$ was in SNNP which could be due to the fact that most the of studies were from this region. Lower recovery rates were recorded in 


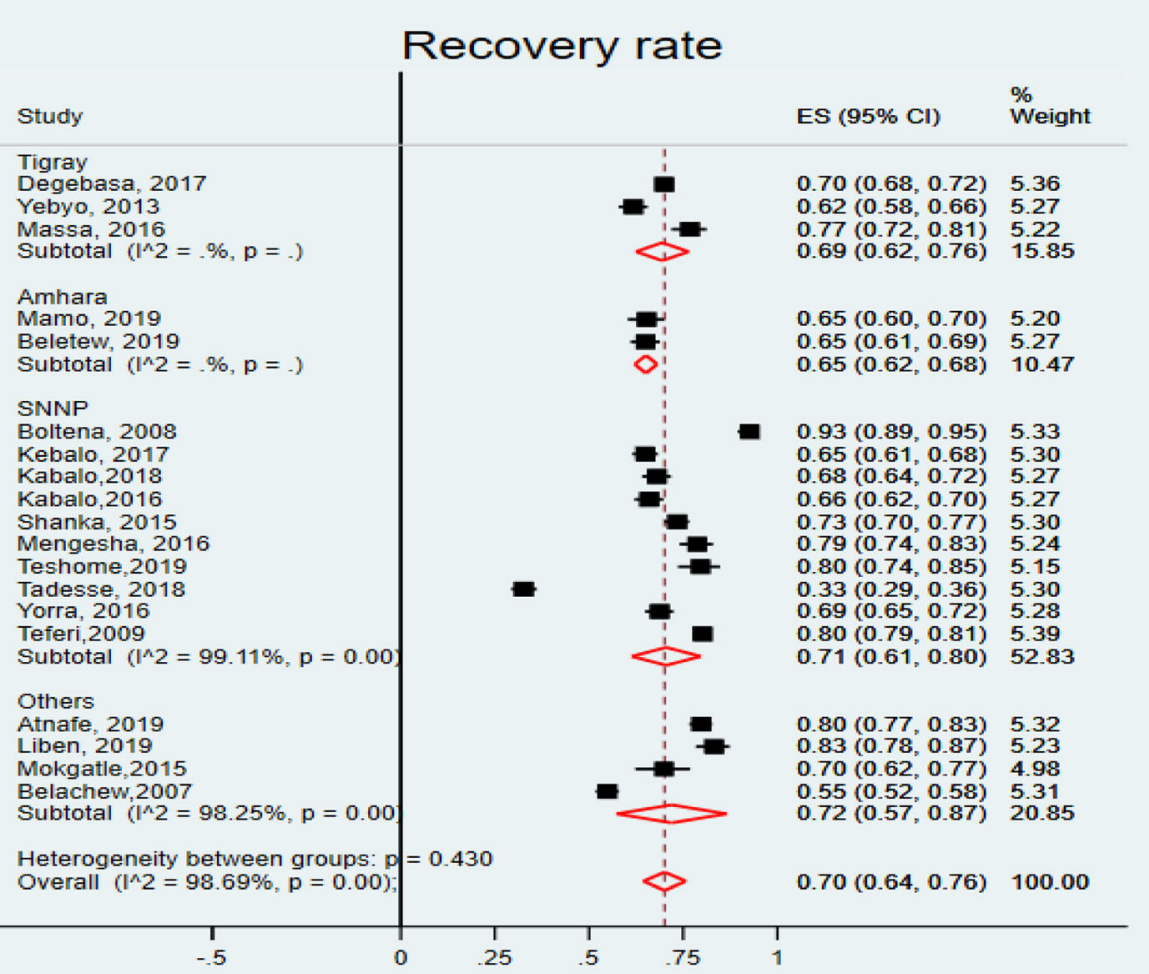

Fig. 2 Forest plot showing the recovery rate of children with SAM treated in OTPs of Ethiopia, 2020

Amhara and Tigray regions. In addition, the recovery rate was computed based on the study settings and children who were treated at health posts $(66,95 \% \mathrm{CI}$ : 54 , $77)$ had poor recovery rates as compared children who were treated in the health centers (71, 95\% CI: 55, 86) (Table 3).

\section{Predictors of recovery rate of SAM children in Ethiopia} In the current review, eight studies [21, 24-26, 31-34] presented the independent predictors of recovery rate using hazard ratios. The predictors which were reported by the original studies were diarrhea [21, 25, 31, 33], age $>24$ months $[26,32,33]$, no edema [31-33], deworming [21, 24, 25, 31, 34] and giving amoxicillin $[21,25,34]$ as part of SAM process. The pooled estimates of hazard ratios revealed that age $>24$ months (HR $=0.98,95 \%$ CI: $\left.0.81,1.15, \mathrm{I}^{2}=80.8, P=0.006\right)$ and deworming (HR $=1.04,95 \% \mathrm{CI}: 0.79,1.28, \mathrm{I}^{2}=43.3, P=$ $0.133)$ were not significantly associated with recovery rate. However, diarrhea, no edema and Amoxicillin were found to be independent predictors of recovery rate (Fig. 7). The recovery rate of SAM children with diarrhea was $16 \%$ less likely compared to SAM children with no diarrhea ( $H R=0.8,95 \%$ CI: $0.75,0.94)$. Similarly, the presence of no edema was found to be a prohibiting factor that decreased recovery rate by $41 \%(\mathrm{HR}=0.41,95 \%$

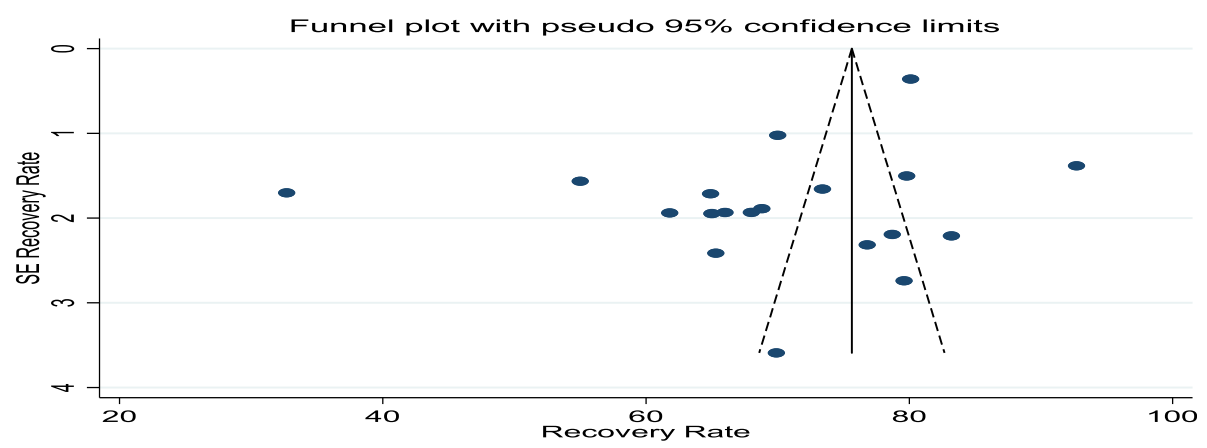

Fig. 3 Funnel plot showing the possible source's bias of recovery rate of children from SAM in OTPs of Ethiopia, 2020 


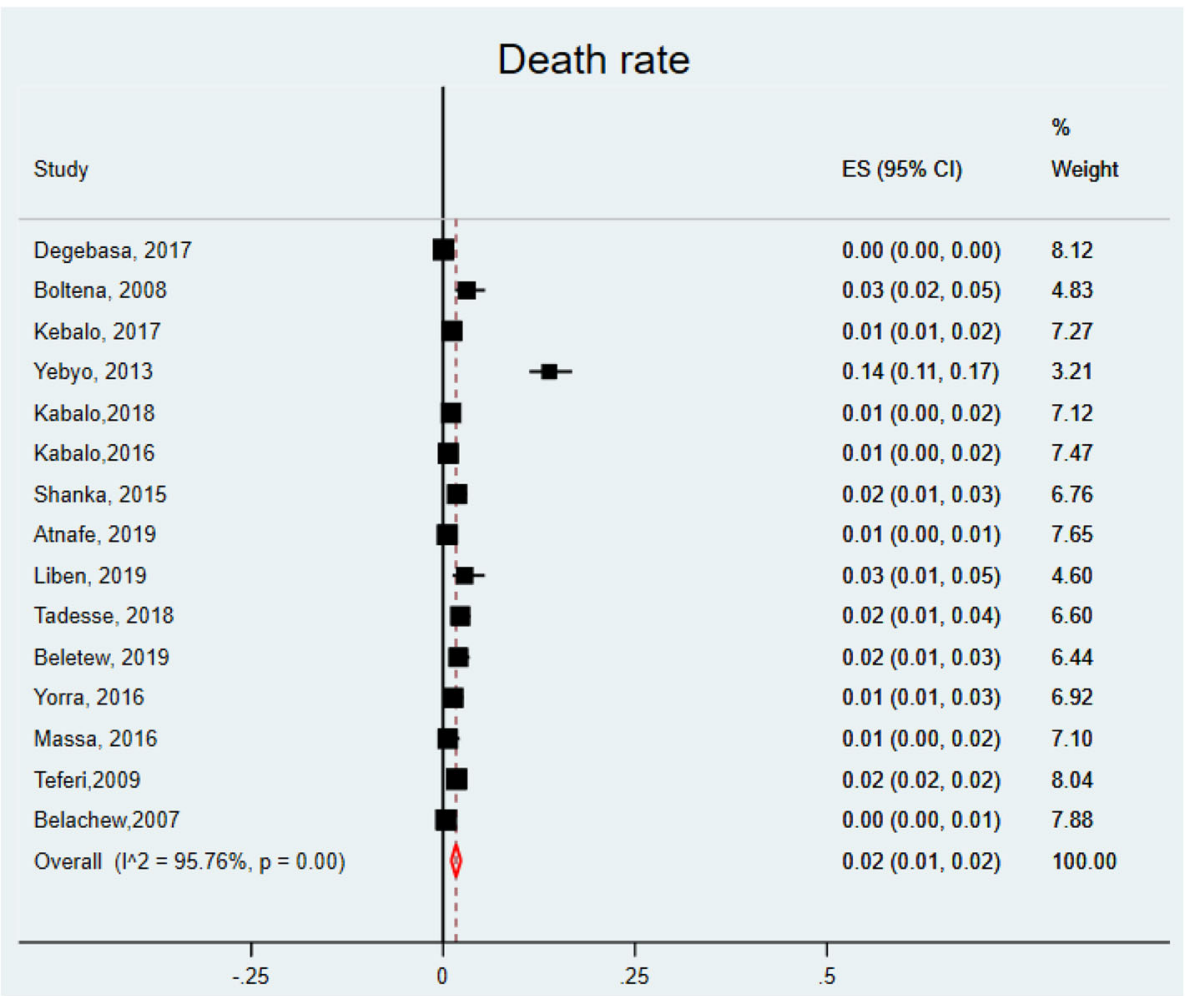

Fig. 4 Forest plot showing the death rate of children with SAM treated in OTPS of Ethiopia, 2020

CI: $0.33,0.50)$. In addition, children who took amoxicillin were nearly two times more likely to recover early from SAM as compared to the counterparts $(\mathrm{HR}=1.81$, 95\% CI:1.18, 2.44).

\section{Discussion}

In this systematic review and meta-analysis, the treatment outcomes of under-five children with SAM admitted in outpatient therapeutic feeding programs of Ethiopia were determined. The treatment outcomes were recovery rate, death rate, defaulter rate and nonrecovery rate. Besides, the predictors of recovery rate were analyzed using hazard ratios as an effect size estimator.

In the current study the proportion of recovery is found to be $70 \%$, which is below the sphere standard, WHO and the national SAM management standards (recovery rate $>75 \%$ ) $[29,46]$. The possible elucidation for the low proportion of recovery could be associated with non-adherence of care givers of children to SAM treatment guidelines. Food sharing, trading of RUTFs as commodity, high cost of standard RUTFs, stigma associated with RUTFs use, lack of antibiotics, inappropriate exit from the program, and disliking the taste of RUTFs could also be the possible rationales for this lower recovery rate $[7,30]$. This finding is comparable with the result of a systematic review about the recovery rate
(70.5\%) [47] of under five children in the inpatient therapeutic feeding programs in Ethiopia but lower than another review with the pooled recovery rate of $72.02 \%$ [48]. This could be due to high comorbidity rates in the inpatient therapeutic feeding programs as compared to the children in the OTPs. This finding is comparable with a study finding in Ghana with the recovery rate of $70.9 \%$ (346 out of 488) [49], but significantly higher than a study finding in Nigeria, where only 58\% (4492 of 7742) of children get cured form SAM [50]. The current recovery rate is also lower than the findings of retrospective studies conducted in Cameroon and Pakistan, where $72.8 \%$ (185 of 254$)$ \& $89 \%(28,882$ of 32,458$)$ of children get recovered, respectively [51, 52]. The discrepancies could be attributed by differences in the number of study population, study design and the sociodemographic characteristics of the study participants as well as variation in the clinical expertise of health care providers.

In the present systematic review and meta-analysis, the pooled estimates of death rate (2\%), defaulting rate $(10 \%)$ and non-recovery rate $(15 \%)$ were determined. The death rate is in line with the sphere and national standards $[29,46]$. Likewise, the current finding is is in line with the death rate reported from Nigeria (2\%) [50]. Nonetheless, this finding is higher than the study findings from Ghana (1.6\%) [49], Cameroon (0.8\%) [52] and 


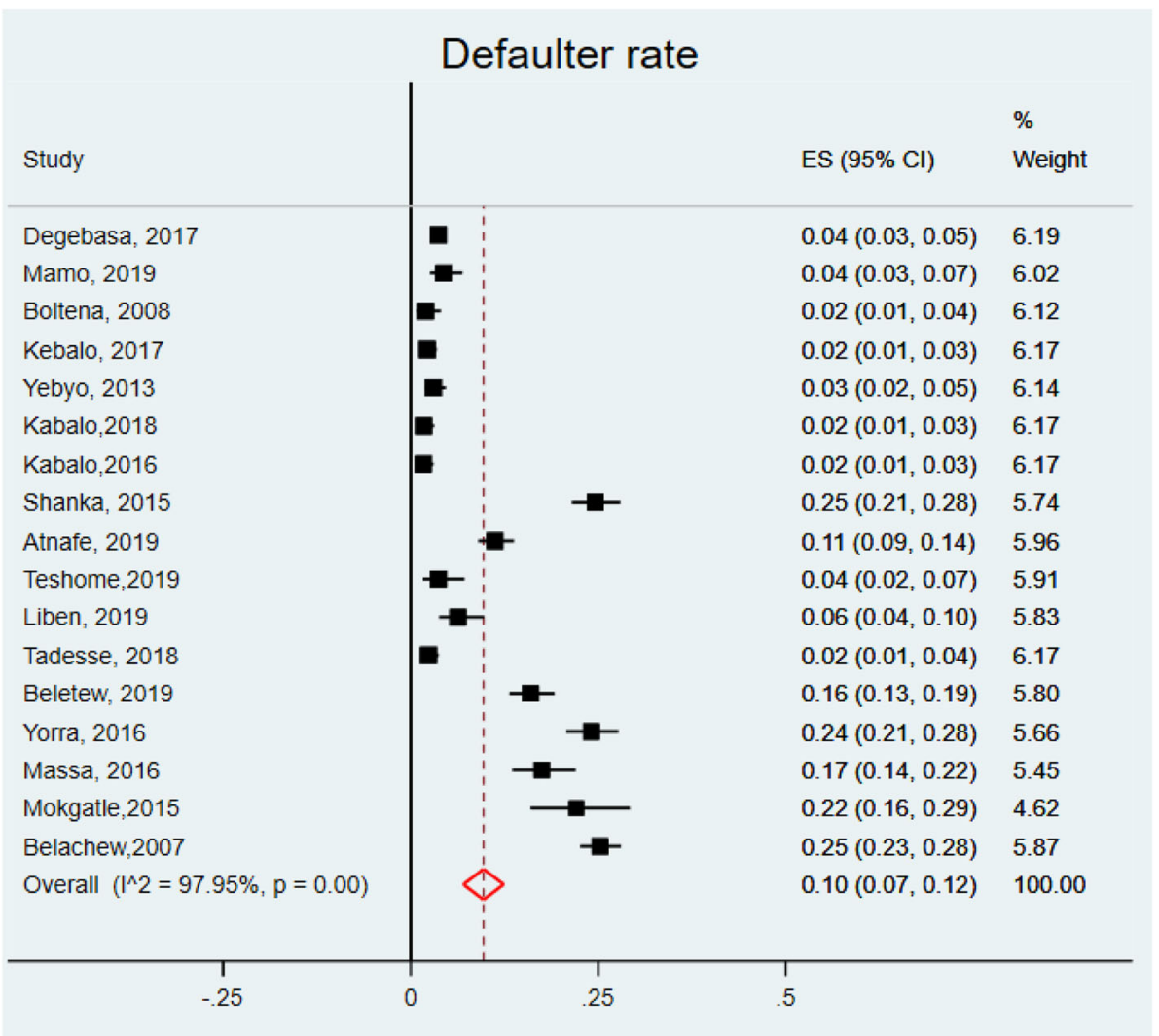

Fig. 5 Forest plot showing the defaulter rate of children with SAM treated in OTPs of Ethiopia, 2020

Pakistan (0.4\%) [51]. The possible explanations for the differences might be due to disparity in the organization of OTP centers, sociodemographic differences in study subjects and difference in the background of care takers of children. Regarding the defaulter rate, this finding coincides with what is recommended by the sphere standard, WHO and national SAM management standards (i.e. defaulting rate $<15 \%)[29,46]$. But the present result is below the findings of the study results in Pakistan [51], Ghana [49] and Nigeria [50] with defaulting rates of 10.6, 28.5 and $40 \%$, respectively. All these findings are from the primary studies and this might be the possible reason for the variations. Differences in the therapeutic areas could also account for the discrepancies. This finding is also significantly lower than the original studies done in different parts of Ethiopia [12, 26-28, 35, 45], of which the proportion of the defaulting range from 16 to $25.2 \%$. Moreover, the proportion of non-recovery (15\%) is considerably higher in this meta-analysis. This is higher than most of the original studies included in this meta-analysis, but lower than the non-recovery rate from the study conducted in Cameroon (26.8\%) [52]. This significant non-recovery rate could be attributed by high burden of comorbidities, inappropriate feeding process of the RUTFs, non-adherence to follow ups due to long distance to access RUTFs and drugs in some OTP centers of the country [7,33-35]. Similarly, food insecurity could contribute to sharing food among family members and this may affect recovery rate of children [33].

Regarding the predictors of recovery rate, the presence of diarrhea, no edema and giving amoxicillin are independent predictors of recovery rate of children. The presence of diarrheal diseases as a comorbidity compromises recovery rate of under-five children by $16 \%$. This is due to the fact that diarrhea and SAM are in vicious cycle. It delays the recovery rate of children with SAM as a result of metabolic disturbances, fluid and electrolyte losses and dehydration. These evidences are supported multitude of studies which implied diarrhea as a major determinant affecting the recovery rate of children with SAM [53-56]. Similarity, children with nonedematous SAM are found have lower recovery rates as compared to the counterparts edematous children. Those children having edema at admission are 59\% more likely to recover within a short duration. This could be substantiated by the likelihood that children with edema might get better care by the health care providers and family members than wasted children [24, 57-59]. Moreover, giving amoxicillin for children with 


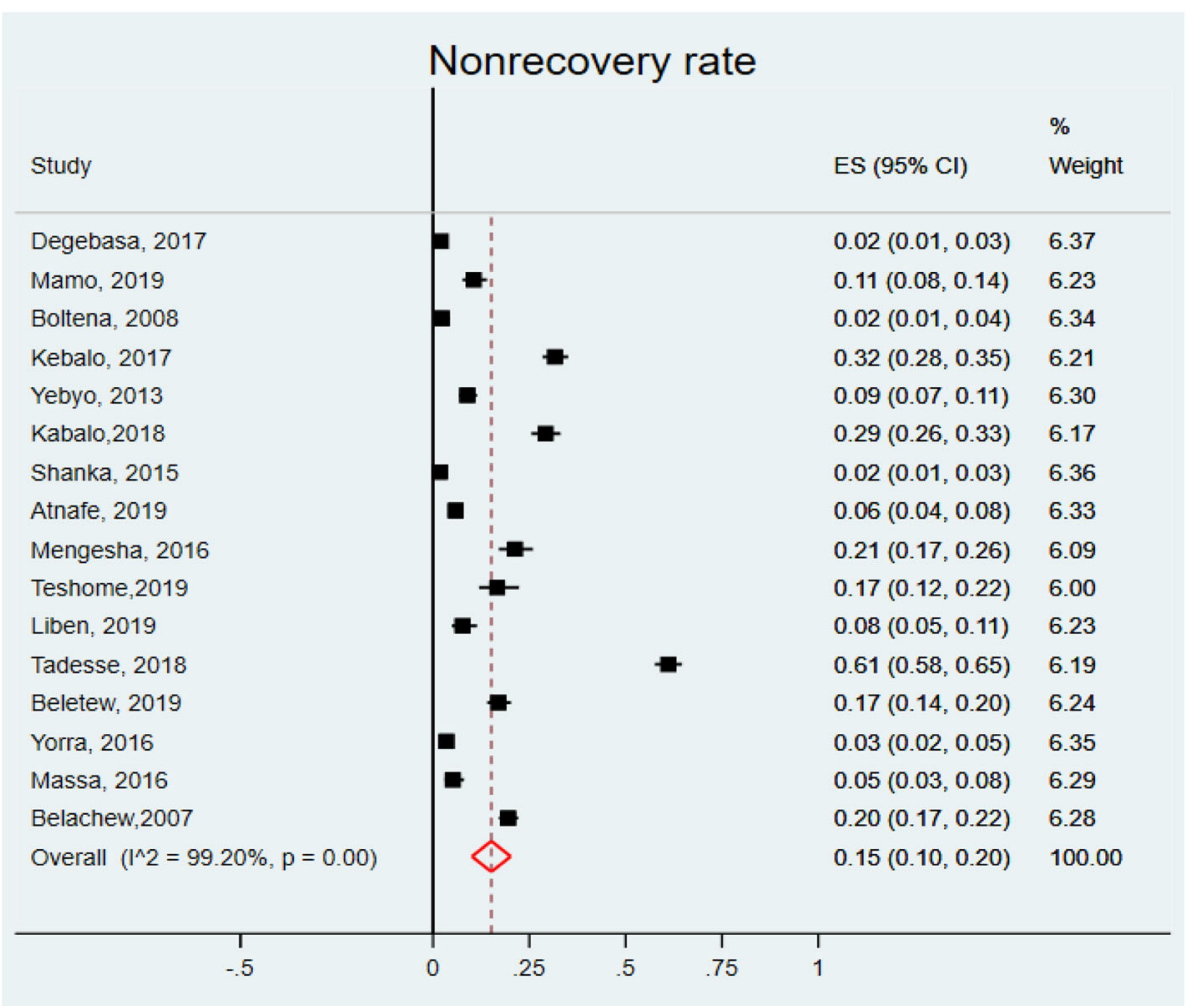

Fig. 6 Forest plot showing the non-recovery rate of children with SAM treated in OTPs of Ethiopia, 2020

SAM as an empirical management is found to enhance survival of children. The likelihood of recovery of children who took amoxicillin is two times compared with children who didn't take it. Severe acute malnutrition affects the whole system and it primarily compromises the immune system of children due to reductive adaption [60]. This paves the way for multiclausal infections which could delay the time of recovery of children [61]. Hence, empirical treatment of infections in the management process of SAM both in the inpatient and outpatient therapeutic feeding programs has a fundamental implication to improve survival of children [62]. Currently, the recommended antibiotic is amoxicillin and this is supported the systematic review and metaanalyses findings which were conducted prior to this systematic review [63, 64].

In general, the current study depicts that the treatment outcomes of children with SAM in OTP centers of Ethiopia is suboptimal. Specifically, the recovery and non-recovery rates are questionable. This could be due to incongruous implementation of the SAM treatment protocols that is corroborated by a systematic review in

Table 3 Subgroup analysis of the recovery rate of under five children with SAM in the outpatient treatment programs of SAM in Ethiopia $(n=19)$

\begin{tabular}{llll}
\hline Variables & Characteristics & Number of studies & Recovery rate $(95 \%$ Cl) \\
\hline Publication year & $\leq 2015$ & 6 & $72 \%(62,82)$ \\
\multirow{3}{*}{ Study Region } & $>2015$ & 13 & $69 \%(62,76)$ \\
& Tigray & 3 & $69 \%(62,76)$ \\
& Amhara & 2 & $65 \%(62,68)$ \\
SNNP & Others & 10 & $71 \%(61,80)$ \\
Study setting & HC & 4 & $72 \%(57,87)$ \\
& HP & 4 & $71 \%(55,86)$ \\
& HC \& HP & 8 & $66 \%(54,77)$ \\
\hline
\end{tabular}




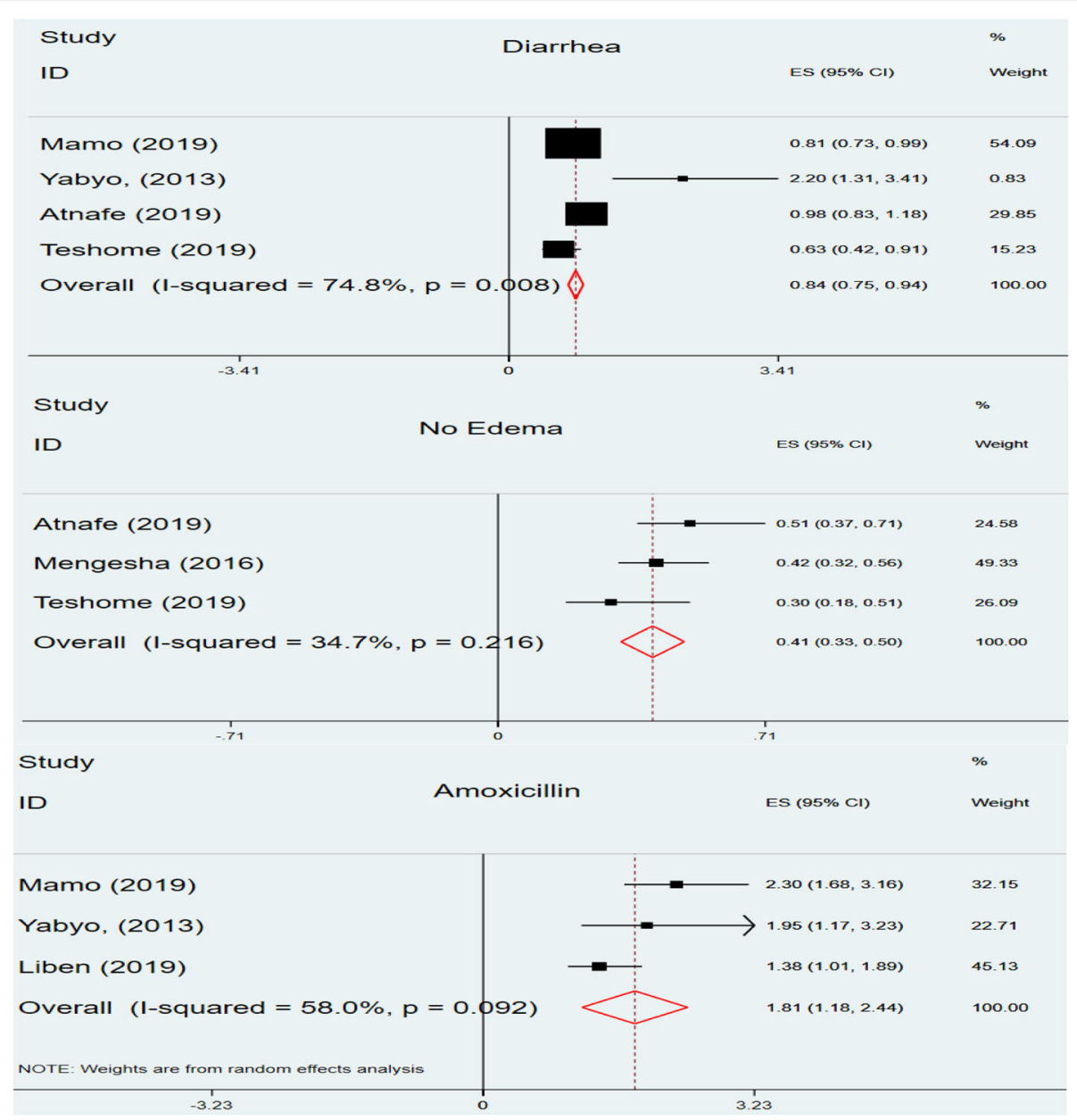

Fig. 7 Forest plot showing predictors of recovery rate among under-five children with SAM in Ethiopia, 2020

which treating SAM children in line with WHO recommendations improved childhood survival [65]. Besides, sharing RUTFs is the main challenge affecting the treatment outcomes of SAM children in Ethiopia [7, 30]. This could be because considerable proportions $(0.9 \%)$ of children and adults have been living with HIV/AIDS that could increase RUTF consumers. Likewise, most of Ethiopian households are inhabited by extended families with the total fertility rate is 4.6 children per woman which perpetuates the food insecurities in the house hold level [66]. Hence, sharing RUTFs among family members could be inevitable.

\section{Strengths and limitations of the study}

To our knowledge, this systematic review and metaanalysis is the first of its type in Ethiopia with so many strengths. The main strength of this systematic review and meta-analysis was that multiples reputable journals were explored comprehensively and exhaustively to retrieve all the original articles. All possible efforts are also made to communicate the primary authors to get articles that were difficult to access the full texts. The data were extracted using standardized and pretested extraction checklist. All possible analyses were done to estimate the pooled treatment outcomes and predictors of recovery rate of children with SAM in OTP centers of Ethiopia. These findings will also help policy makers, stakeholders, nongovernmental organizations and community health workers to modify their approach in the management process of children in OTPs. Despite these strengths, the current study has some limitations. Only articles that were published in the English language were included in this meta-analysis, which might affect the true estimates of treatment outcomes. To estimate the pooled predictors, limited numbers of studies were obtained and this might be the cause for under estimation predictors of recovery rates. In addition, the predictors were calculated using hazard ratios, but some studies reported factors using odds ratios. But none of the variables were found to have significant association with recovery. This could compromise the number of independent predictors. 


\section{Conclusion}

This meta-analysis revealed that the proportion of recovery and non-recovery were significantly higher than the sphere standard, WHO and national SAM management protocols. This finding is comparable with the recovery rate form inpatient units of the country which should not be really the case. This finding is alarming for policy makers and program implementers in Ethiopia. The presence of diarrheal disease as comorbidity and being non-edematous at admission were found to be prohibiting factors of time to recovery of children with SAM. In contrary, empirical treatment of children with amoxicillin was found to shorten the duration of recovery from SAM. Policy makers, community health workers, and program planners need to reconsider the community based management approaches of children with SAM.

\section{Supplementary information}

Supplementary information accompanies this paper at https://doi.org/10. 1186/s12887-020-02188-5.

Additional file 1. Critical Appraisal.

Additional file 2. PRISMA Checklist.

Additional file 3. Search String.

\section{Abbreviations}

Cl: Confidence interval; CMAM: Community based management of acute malnutrition; HR: Hazard ratio; OTP: Outpatient therapeutic feeding program; RUTF: Ready to use therapeutic food; SAM: Severe acute malnutrition; SNNP: Southern national, nationalities and peoples in Ethiopia; WHO: World health organization

\section{Acknowledgments}

The authors acknowledge all the authors the original studies included in this systematic review and meta-analysis.

\section{Authors' contributions}

ZBW, TW and AA conceived and designed the review. ZWB prepared the draft of the manuscript. The final version of the manuscript is approved by all the three authors

\section{Funding}

No fund is incurred for this systematic review and meta-analysis.

\section{Availability of data and materials}

All-important data for this study are included in the manuscript. If in need of additional data, they can be accessed from the corresponding author.

\section{Ethics approval and consent to participate}

Not applicable for this study.

\section{Consent for publication}

Not applicable.

\section{Competing interests}

The authors have declared no conflicts of interest in this work.

\section{Author details}

${ }^{1}$ Department of Pediatric Nursing, St. Paul's Hospital Millennium Medical College, School of Nursing, Addis Ababa, Ethiopia. ${ }^{2}$ Ethipian Public Health Institute, Addis Ababa, Ethiopia. ${ }^{3}$ College of Health and Medical Sciences, School of Nursing and Midwifery, Haramaya University, Harar, Ethiopia.
Received: 18 February 2020 Accepted: 2 June 2020

Published online: 07 July 2020

\section{References}

1. Golden M, Grellety Y. Guidelines for the integrated management of severe acute malnutrition: in-and out-patient treatment. Paris: ACF International; 2011

2. Hayashi C, Krasevec J, Kumapley R, Mehra V, de Onis M, Borghi E, et al. Levels and trends in child malnutrition. UNICEF/WHO/World Bank Group joint child malnutrition estimates: key findings of the 2017 edition. 2017.

3. Shoham J, McGrath M. Editorial perspective on the continuum of care for children with acute malnutrition. Field Exchange. 2019;60:p2.

4. Unicef. THE STATE OF WORLD'S CHILDREN 2019, Children, Food and Nutrition 2019.

5. Olofin I, McDonald CM, Ezzati M, Flaxman S, Black RE, Fawzi WW, et al. Associations of suboptimal growth with all-cause and cause-specific mortality in children under five years: a pooled analysis of ten prospective studies. PloS One. 2013;8(5):e64636.

6. Bhutta ZA, Berkley JA, Bandsma RH, Kerac M, Trehan I, Briend A. Severe childhood malnutrition. Nat Rev Dis Primers. 2017:3:17067.

7. Tadesse E, Ekström E-C, Berhane Y. Challenges in implementing the integrated community-based outpatient therapeutic program for severely malnourished children in rural southern Ethiopia. Nutrients. 2016;8(5):251.

8. Teferi E, Lera M, Sita S, Bogale Z, Datiko DG, Yassin MA. Treatment outcome of children with severe acute malnutrition admitted to therapeutic feeding centers in Southern Region of Ethiopia. Ethiopian J Health Dev. 2010; 24(3)234-8.

9. UNICEF. Evaluation of community management of acute malnutrition (CMAM). Ethiopia: Federal Ministry of Health, Government of Ethiopia and UNICEF Country Office; 2012.

10. Golden M, Grellety Y. Protocol for the management of severe acute malnutrition. Ethiopia: $\mathrm{MOH} ; 2007$.

11. Organization WH, UNICEF. Community-based management of severe acute malnutrition: a joint statement by the World Health Organization, the World Food Programme, the United Nations System Standing Committee on Nutrition and the United Nations Children's Fund. Community-based management of severe acute malnutrition: a joint statement by the World Health Organization, the World Food Programme, the United Nations System Standing Committee on Nutrition and the United Nations Children's Fund. 2007

12. Belachew T, Nekatibeb H. Assessment of outpatient therapeutic programme for severe acture malnutrition in three regions of Ethiopia. East Afr Med J. 2007:84(12):577-88.

13. Collins S. Changing the way we address severe malnutrition during famine. Lancet. 2001;358(9280):498-501.

14. Bhutta ZA, Berkley JA, Bandsma RH, Kerac M, Trehan I, Briend A. Severe childhood malnutrition. Nat Rev Dis Primers. 2017;3(1):1-18.

15. Joseph Kawuki1* THM, $3^{*}$, Upama Ghimire1, Nathan Obore1 and Shireen Salome Papabathini1. Community-Based Management of Acute Malnutrition: An Effective Intervention for Under-Nutrition Journal of Complementary Medicine \& Alternative Healthcare. 2019;10(4):555791. https://doi.org/10.19080/JCMAH.2019.10.555791.

16. Tekeste A, Wondafrash M, Azene G, Deribe K. Cost effectiveness of community-based and in-patient therapeutic feeding programs to treat severe acute malnutrition in Ethiopia. Cost Effectiveness Resource Allocation. 2012;10(1):4.

17. Bachmann MO. Cost-effectiveness of community-based treatment of severe acute malnutrition in children. Expert Rev Pharmacoecon Outcomes Res. 2010;10(5):605-12.

18. Tadesse E, Worku A, Berhane Y, Ekström EC. An integrated communitybased outpatient therapeutic feeding programme for severe acute malnutrition in rural southern Ethiopia: recovery, fatality, and nutritional status after discharge. Maternal Child Nutr. 2018;14(2):e12519.

19. Boltena SS. Factors affecting the rehabilitation outcome (of outpatient therapeutic program) of children with severe acute malnutrition in Durame. Southern Ethiopia: UWC; 2008.

20. Degebasa Z, Marama T, Addisu A. Magnitude and treatment outcome of severe acute malnutrition of 6-59 months children in Raya Alamata Woreda, Tigray Region, Ethiopia from 2011-2015. Science (New York, NY). 2017;5(5-1):30-7. 
21. Mamo WN, Derso T, Gelaye KA, Akalu TY. Time to recovery and determinants of severe acute malnutrition among 6-59 months children treated at outpatient therapeutic programme in North Gondar zone, Northwest Ethiopia: a prospective follow up study. Ital J Pediatr. 2019;45(1): 136.

22. Kabalo MY, Seifu CN. Treatment outcomes of severe acute malnutrition in children treated within Outpatient Therapeutic Program (OTP) at Wolaita Zone, Southern Ethiopia: retrospective cross-sectional study. J Health Popul Nutr. 2017;36(1):7.

23. Kabalo MY, Shanka MM. Seasonal variations of admission and survival status of children treated for severe acute malnutrition (SAM) at outpatient therapeutic program (OTP) in Wolaita zone, southern Ethiopia. Int J Collaborative Res Internal Med Public Health. 2016;8(9):512-26.

24. Kabalo MY, Yohannes B. Children with oedema recover better than those with severe wasting in outpatient therapeutic program at Boloso sore district, Southwest Ethiopia. BMC Research Notes. 2018;11(1):118.

25. Yebyo HG, Kendall C, Nigusse D, Lemma W. Outpatient therapeutic feeding program outcomes and determinants in treatment of severe acute malnutrition in Tigray, northern Ethiopia: a retrospective cohort study. PLoS One. 2013;8(6):e65840.

26. Shanka N, Lemma S, Abyu D. Recovery rate and determinants in treatment of children with severe acute malnutrition using outpatient therapeutic feeding program in Kamba District, south West Ethiopia. J Nutr Disord Ther. 2015;5(2):155.

27. Beletew B, Deresse B, Mengesha A, Wudu M. Biruk Beletew. Treatment Outcome of Severe Acute Malnutrition and associated factors among under-five children in outpatient therapeutics unit in Gubalafto Wereda, North Wollo Zone, Amara Regional State, North Ethiopia. 2019. G.C. BMC Research Square: https://doi.org/10.21203/rs.2.16242/v1

28. MOKGATLE M, DEMISSE B. Community-based management programme for treatment of acute child malnutrition using the out-patient therapeutic treatment approach in Dhas district of Ethiopia. African J Phys Health Educ Recreation Dance. 2015;21(Supplement 2):39-49.

29. Association S. Sphere handbook: humanitarian charter and minimum standards in humanitarian response: PRACTICAL ACTION; 2018.

30. Ersino G, Whiting SJ, Henry CJ. Ready-to-use therapeutic and supplementary foods in Ethiopia from 2006-2018: scoping review. Int J Clin Nutr Dietetics. 2018;2018(4):138

31. Atnafe $B$, Roba KT, Dingeta T. Time of recovery and associated factors of children with severe acute malnutrition treated at outpatient therapeutic feeding program in Dire Dawa, Eastern Ethiopia. PloS One. 2019;14(6):e0217344.

32. Mengesha MM, Deyessa N, Tegegne BS, Dessie Y. Treatment outcome and factors affecting time to recovery in children with severe acute malnutrition treated at outpatient therapeutic care program. Glob Health Action. 2016; 9(1):30704.

33. Teshome G, Bosha T, Gebremedhin S. Time-to-recovery from severe acute malnutrition in children 6-59 months of age enrolled in the outpatient treatment program in Shebedino, southern Ethiopia: a prospective cohort study. BMC Pediatr. 2019;19(1):33.

34. Liben ML, Wuneh AG, Shamie R. Factors associated with child survival in children admitted to outpatient therapeutic program at public health institutions in Afar Regional State, Ethiopia: a prospective cohort study. J Health Popul Nutr. 2019;38(1):35.

35. Massa D, Woldemichael K, Tsehayneh B, Tesfay A. Treatment outcome of severe acute malnutrition and determinants of survival in northern Ethiopia: a prospective cohort study. Int J Nutr Metab. 2016;8(3):12-23.

36. Moher D, Liberati A, Tetzlaff J, Altman DG. Preferred reporting items for systematic reviews and meta-analyses: the PRISMA statement. Ann Intern Med. 2009;151(4):264-9.

37. Munn ZMS, Lisy K, et al. Methodological guidance for systematic reviews of observational epidemiological studies reporting prevalence and cumulative incidence data. Int J Evid Based Healthc. 2015;13:147-53.

38. Sterne JA, Egger M. Funnel plots for detecting bias in meta-analysis: guidelines on choice of axis. J Clin Epidemiol. 2001;54(10):1046-55.

39. Rücker G, Schwarzer G, Carpenter JR, Schumacher M. Undue reliance on 12 in assessing heterogeneity may mislead. BMC Med Res Methodol. 2008:8(1):79.

40. Higgins JP, Thompson SG. Quantifying heterogeneity in a meta-analysis. Statistics Med. 2002;21(11):1539-58.

41. DerSimonian R, Laird N. Meta-analysis in clinical trials. Control Clin Trials. 1986;7(3):177-88.
42. Borenstein $M$, Hedges LV, Higgins JP, Rothstein HR. A basic introduction to fixed-effect and random-effects models for meta-analysis. Res Synth Methods. 2010;1(2):97-111.

43. Begg CB, Mazumdar M. Operating characteristics of a rank correlation test for publication bias. Biometrics. 1994;50:1088-101.

44. Teferi E, Teklemariam S, Erosie L, Hailu A, Belachew T, Yassin M. Management of severe acute malnutrition in children using community based therapeutic care approach: a review of three years data from southern Ethiopia. Ethiopian J Pediatrics Child Health. 2009;5(5):49-59.

45. Yorra DT, Sagar G. Survival rate and determinants in treatment of children with severe acute malnutrition using outpatient therapeutic feeding program in Sidama zone. South Ethiopia IOSR J Mathematics. 2016:12:86-100

46. Federal democratic Republic of Ethiopia MoH. TRAINING COURSE ON THE MANAGEMENT OF SEVERE ACUTE MALNUTRITION. 2013.

47. Wagnew F, Dessie G, Takele WW, Tadesse A, Islam SMS, Mulugeta H, et al. A meta-analysis of inpatient treatment outcomes of severe acute malnutrition and predictors of mortality among under-five children in Ethiopia. BMC Public Health. 2019;19(1):1-11.

48. Yazew KG, Kassahun CW, Ewnetie AW, Mekonen HK, Abagez ES. Recovery rate and determinants of severe acute malnutrition children treatment in Ethiopia: a systematic review and meta-analysis. Systematic Rev. 2019;8(1):1-7.

49. Akparibo R, Harris J, Blank L, Campbell MJ, Holdsworth M. Severe acute malnutrition in children aged under 5 years can be successfully managed in a non-emergency routine community healthcare setting in Ghana. Maternal Child Nutr. 2017;13(4):e12417.

50. Farouk ZL, Gwarzo GD, Zango A, Abdu H. Improving the outcome of severe acute malnutrition by community-based management. J Health Res Rev. 2016;3(1):20.

51. Aguayo VM, Badgaiyan N, Qadir SS, Bugti AN, Alam MM, Nishtar N, et al. Community management of acute malnutrition (CMAM) programme in $\mathrm{P}$ akistan effectively treats children with uncomplicated severe wasting. Maternal Child Nutr. 2018;14:e12623.

52. Ndzo JA, Jackson A. Outcomes of children aged 6-59 months with severe acute malnutrition at the GADO outpatient therapeutic Center in Cameroon. BMC research notes. 2018;11(1):68.

53. Talbert A, Thuo N, Karisa J, Chesaro C, Ohuma E, Ignas J, et al. Diarrhoea complicating severe acute malnutrition in Kenyan children: a prospective descriptive study of risk factors and outcome. PloS one. 2012;7(6):e38321.

54. Irena $\mathrm{AH}$, Mwambazi M, Mulenga V. Diarrhea is a major killer of children with severe acute malnutrition admitted to inpatient set-up in Lusaka. Zambia Nutrition journal. 2011;10:110.

55. Grenov B, Lanyero B, Nabukeera-Barungi N, Namusoke H, Ritz C, Friis $H_{\text {, }}$ et al. Diarrhea, dehydration, and the associated mortality in children with complicated severe acute malnutrition: a prospective cohort study in Uganda. J Pediatr. 2019;210:26-33.e3. https://doi.org/10.1016/j.jpeds.2019.03. 014.

56. Manary M, lannotti L, Trehan I, Weisz A. Systematic review of the care of children with diarrhoea in the community-based management of severe acute malnutrition. Washington: WHO; 2012.

57. Gebremichael DY. Predictors of nutritional recovery time and survival status among children with severe acute malnutrition who have been managed in therapeutic feeding centers, southern Ethiopia: retrospective cohort study. BMC Public Health. 2015;15(1):1267.

58. Saaka M, Osman SM, Amponsem A, Ziem JB, Abdul-Mumin A, Akanbong P, et al. Treatment outcome of severe acute malnutrition cases at the tamale teaching hospital. J Nutr Metab. 2015;2015:641784.

59. Faurholt-Jepsen $D$, Hansen KB, van Hees VT, Christensen LB, Girma T, Friis $H$, et al. Children treated for severe acute malnutrition experience a rapid increase in physical activity a few days after admission. J Pediatr. 2014; 164(6):1421-4.

60. Cloete J. Management of severe acute malnutrition. S Afr Med J. 2015; 105(7):605.

61. Bwakura-Dangarembizi M, Amadi B, Bourke CD, Robertson RC, Mwapenya B, Chandwe $\mathrm{K}$, et al. Health outcomes, pathogenesis and epidemiology of severe acute malnutrition (HOPE-SAM): rationale and methods of a longitudinal observational study. BMJ Open. 2019;9(1):e023077.

62. Trehan I, Goldbach HS, LaGrone LN, Meuli GJ, Wang RJ, Maleta KM, et al. Research article (New England journal of medicine) antibiotics as part of the management of severe acute malnutrition. Malawi Med J. 2016;28(3):123-30. 
63. Williams PC, Berkley JA. Guidelines for the treatment of severe acute malnutrition: a systematic review of the evidence for antimicrobial therapy. Paediatrics Int Child Health. 2018;38(sup1):S32-49.

64. Alcoba G, Kerac M, Breysse S, Salpeteur C, Galetto-Lacour A, Briend A, et al. Do children with uncomplicated severe acute malnutrition need antibiotics? A systematic review and meta-analysis. PloS One. 2013;8(1):e53184.

65. Tickell KD, Denno DM. Efficacy of World Health Organization Guideline in Facility-Based Reduction of Mortality in Severely Malnourished Children From Low and Middle Income Countries. J Paediatrics Child Health. 2017; 53(9):925.

66. EDHS E. Demographic and health survey 2016: key indicators report. The DHS Program ICF. 2016.

\section{Publisher's Note}

Springer Nature remains neutral with regard to jurisdictional claims in published maps and institutional affiliations.

Ready to submit your research? Choose BMC and benefit from:

- fast, convenient online submission

- thorough peer review by experienced researchers in your field

- rapid publication on acceptance

- support for research data, including large and complex data types

- gold Open Access which fosters wider collaboration and increased citations

- maximum visibility for your research: over $100 \mathrm{M}$ website views per year

At BMC, research is always in progress.

Learn more biomedcentral.com/submissions 